\section{The right sort of roughness}

\section{Peter Carpenter}

Skin-friction drag affects any surface moving through a fluid. But a new theory of near-wall turbulence has led to a strange new drag-reduction technique: applying a random arrangement of bumps.

E very surfer believes that polished boards create less drag. This view has always been somewhat at odds with the natural world, where smooth surfaces are rare. It was further undermined with the discovery by the US space agency NASA ${ }^{1}$, and by scientists studying the hydrodynamic effects of shark scales ${ }^{2}$, that surfaces with riblets minute streamwise grooves - experience lower drag than smooth ones. The 'smooth is best' precept has now received a further setback. On page 753 of this issue ${ }^{3}$, Sirovich and Karlsson show that surfaces covered with special randomized patterns of small vshaped protuberances produce lower drag than smooth ones. But regular patterns of the same protuberances lead to a rise in drag, so the surfers are not completely wrong!

The type of drag in question is skin-friction drag, which is caused by the action of viscous shear forces on the surface. It is much greater when the flow is turbulent, as in most engineering applications. Drag reduction has been an obsession of practical aerodynamicists for most of the twentieth century; equally, achieving a theoretical understanding of turbulence has remained the elusive goal of many of the finest minds during the same period. Hitherto, the discovery of new dragreduction methods has done far more to advance the theories of turbulence than vice versa. In this respect Sirovich and Karlsson are a rare exception, because their drag-reduction method is the fruit of their efforts to develop a theoretical understanding of the turbulent air flow in a two-dimensional channel.

The turbulent flow near a channel wall is bewilderingly complex. This complexity is inherent in the flow physics, and stems, fundamentally, from Newton's laws of motion. Turbulence is found when the effects of viscosity are weak - it is an intriguing paradox of fluid mechanics that the weaker the direct action of viscosity the more complex its effects. Most authorities now agree that deterministic structures (usually termed coherent structures), which are predictable in their general form, are created in turbulent flow. But these coherent structures have a broad, random distribution in time and space, and they are characterized by a very wide range of length and timescales.

It is generally believed that the crucial dynamics occur in a thin, highly sheared, region, extending up to $2 \mathrm{~mm}$ from the wall in the channel-flow experiment of Sirovich and Karlsson. A veritable zoo of coherent structures has been identified by successive experimentalists, and a common view of the dynamics has emerged (Fig.1). Low-speed streaks appear near the wall ${ }^{4}$, caused by streamwise vortices. As these vortices develop they lift up away from the surface ('ejection') and finally 'burst'. Bursting generates intense turbulence and appears to initiate the formation of new vortex structures.

The key questions are, what causes ejection and bursting, and how does bursting create new vortices? There are many different theoretical models. For example, Smith et $a l .{ }^{5}$ believe that the bursts occur when a vortex approaches the wall, causing strong local flow deceleration and subsequent flow separation (eruption) from the wall, thereby triggering the birth of a new vortex. On the other hand, Sirovich and his co-workers believe that, although the vortices are the primary structures, there are also much weaker oblique plane waves propagating downstream, and that bursting and rebirth come about through an interaction between a pair of waves and the vortices. In previous computer simulations they found that artificially randomizing the phases of the vortices and oblique waves led to less bursting and consequently to a reduction in drag.

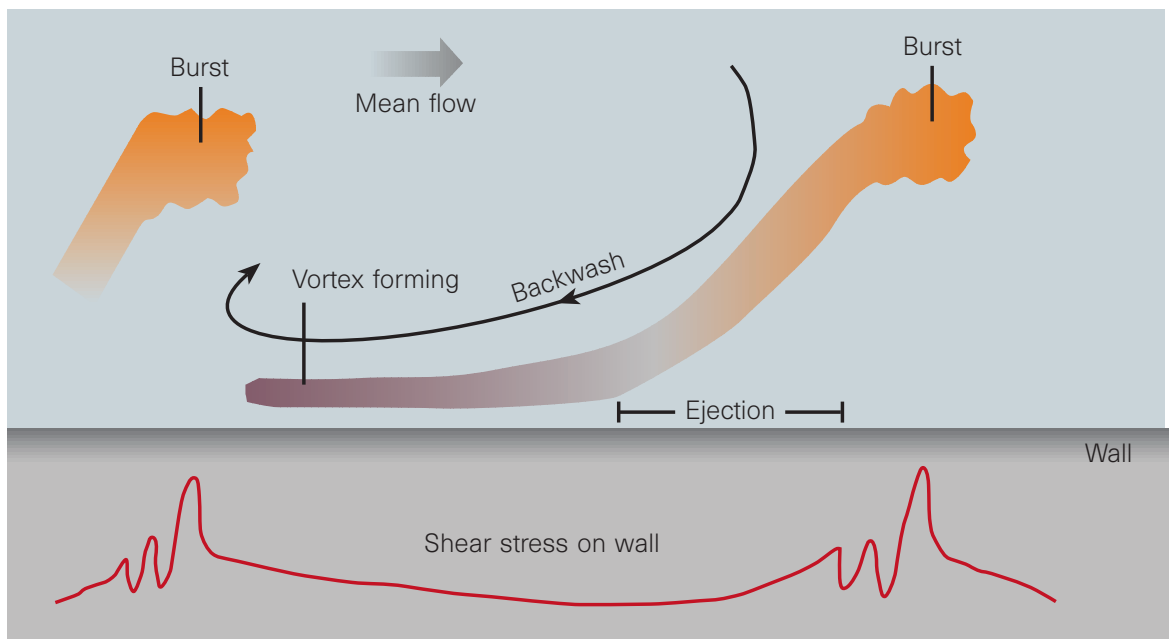

Figure 1 Simplified side view of near-wall turbulence. Most of the shear stress (or drag) is created where vortices 'burst' away from the wall. An unproved idea is that bursting is caused by an interaction between the vortices and pairs of oblique plane waves. It has led to an effective new method of drag reduction ${ }^{3}$ : randomized bumps that break up the phase coherence of the vortices. 
running costs than fuel), or interfere with maintenance, either compromising safety or increasing costs.

For these reasons, riblets have not yet been used for commercial aircraft; and the new method would probably be no more attractive. However, it may have advantages for other engineering applications, for example high-speed sailing yachts, such as America's Cup competitors, for which skin friction contributes more than half the total drag.
Peter Carpenter is in the Department of

Engineering, University of Warwick, Coventry CV4 7AL, UK.

1. Walsh, M. J. Am. Inst. Aeronautics and Astronautics Paper 820169 (1982).

2. Bechert, D. W., Hoppe, G. \& Reif, W.-E. Am. Inst. Aeronautics and Astronautics Paper 85-0546 (1985).

3. Sirovich, L. \& Karlsson, S. Nature 388, 753-755 (1997).

4. Kline, S. J., Reynolds, W. C., Schraub, F. A. \& Runstadler, P. W. J. Fluid Mech. 95, 741-773 (1967).

5. Smith, C. R., Walker, J. D. A., Haidari, A. H. \& Sobrun, U. Phil. Trans. R. Soc. Lond. A 336, 131-175 (1991).

\section{CED-4 is a stranger no more}

Michael O. Hengartner

T he nematode worm Caenorhabditis elegans has been used with great success to identify the basic components of the machinery underlying apoptosis (programmed cell death $)^{1}$. Indeed, of the three key cell death genes that have been identified in C. elegans, two - ced-3 and ced-9 - have mammalian homologues that also function in apoptosis. But the sequence of the third gene, $c e d-4$, revealed no obvious mammalian homologue, and precious little in terms of possible mechanism of action. A flurry of activity has changed that. A paper by Zou et al., published earlier this month in Cell ${ }^{2}$, provides a homologue. And work by Chinnaiyan et al. (page 728 of this issue ${ }^{3}$ ) and by Seshagiri and Miller in Current Biology $y^{4}$ lays down some choreography for the part that CED-4 protein plays in the molecular dance of death.

Over the years, genetic screens in C. elegans have led to the identification of about a dozen cell death (ced) genes that are responsible for one aspect or another of the apoptotic process. Three of these genes stand out. Two, ced-3 and ced-4, are essential for cell death. The third, ced-9, antagonizes the proapoptotic activities of ced-3 and ced-4, and thereby protects cells that should survive from any accidental activation of the death programme.

Real stardom for ced-9 and ced-3 only came with their cloning, when it became clear that they encode components of a universal and highly conserved death machinery: CED-9 protein turned out to be a member of the Bcl-2 family of cell death regulators, whereas CED-3 was homologous to a family of proapoptotic cysteine proteases, known as the caspases. Indeed, identification of CED-3 as a caspase homologue was the first evidence of this family's involvement in apoptosis. In both worm and human, CED-9/Bcl-2 act upstream of the 'execution caspases' such as CED-3 and caspase-3, somehow preventing their proteolytic processing from zymogens into fully active killer enzymes. But how Bcl-2 et al. perform this feat remains very much up in the air.

The ced-4 story was initially much less glamorous. Because the gene encoded a pre- viously unknown protein without any mammalian homologue, its cloning did not provide much help in explaining how it might promote cell death. Genetically, ced-4 had been placed between ced-9 and ced-3 in the pathway leading to cell death ${ }^{5}$, suggesting that it might act as an adaptor, linking the upstream regulator CED-9 to the downstream death effector CED-3.

Strong experimental support for this view came from four papers published early this year, which collectively showed that CED-4 can interact directly and simultaneously with both CED-9 and CED-3 (reviewed in refs 6, 7); the interaction must be quite stable, as CED-4 and CED-9 colocalize when co-expressed. These observations point to a model in which CED-9 prevents cell death by directly binding to a CED4/CED-3 complex, presumably keeping it in

a

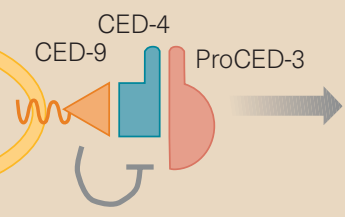

b

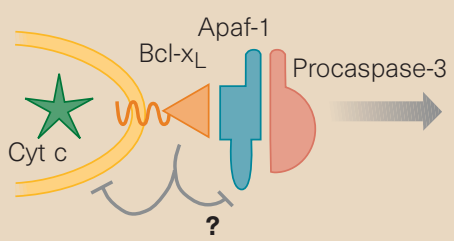

Figure 1 Caspases are cysteine proteases that promote apoptosis in mammals and have homologues in C. elegans. a and b depict highly simplified schemes of how they might be activated in the two systems. a, In C. elegans the inactive CED-9/4/3 complex is bound to cell membranes by CED-9's hydrophobic tail. On reception of a death-inducing stimulus, something happens (here shown as the physical separation of CED-4/3 from CED-9) which allows processing and activation of the 'execution caspase' CED-3. $\mathrm{b}$, In mammals, the Bcl-2 family might perform double duty, preventing cytochrome $c$ from leaving mitochondria and also possibly binding to Apaf-1 (one of the so-called apoptosis protease-activating factors, of which cytochrome $c$ is Apaf-2). The death-inducing stimulus would allow formation of the full Apaf complex, possibly including Apaf-3, leading to caspase-3 processing and activation. Question marks point to aspects that are even more egregiously speculative than the rest of the model. 